\title{
Diisopropyl L-Malate as a New Chiral Auxiliary for Dynamic Kinetic Resolution of $\alpha$-Bromo Esters and Asymmetric Syntheses of Aminoflavones and Dihydroquinoxalinones
}

\author{
Kyoung Hee Kang, Jung In Jang, Seung Bin Baek, Jung Hee Ahn, and Yong Sun Park* \\ Department of Chemistry and Bio/Molecular Informatics Center, Konkuk University, Seoul 143-701, Korea \\ ${ }^{*}$ E-mail: parkyong@konkuk.ac.kr \\ Received February 28, 2011, Accepted March 7, 2011
}

Key Words : Asymmetric synthesis, Chiral auxiliary, Dynamic kinetic resolution, Nucleophilic substitution, Flavonoids

Malic acid is the active ingredient in many sour foods and found mostly in unripe fruits. The dicarboxylic acid is relatively inexpensive and commercially available in both enantiomeric forms. The application of malic acid derivatives as a chiral auxiliary is, however, not well known in the area of asymmetric synthesis. ${ }^{1}$ We herein report the first example of L-malate-mediated dynamic kinetic resolution of $\alpha$ bromo esters in nucleophilic substitution with various arylamines. $^{2}$

Treatment of diisopropyl L-malate with racemic $\alpha$-bromo phenylacetic acid in the presence of DCC and DMAP provided $\alpha$-bromo phenylacetate $(\alpha R S)-1$ in $77 \%$ yield with about 50:50 diastereomeric ratio (dr). When the two diastereomeric mixture of $(\alpha R S)-\mathbf{1}$ was treated with $p$-anisidine (1.5 equiv), tetrabutylammonium iodide (TBAI, 1.0 equiv) and diisopropylethylamine (DIEA, 1.0 equiv) in $\mathrm{CH}_{2} \mathrm{Cl}_{2}$ at room temperature for $12 \mathrm{~h}, \mathrm{~N}$-aryl amino ester 2 was produced in $81 \%$ yield with 90:10 dr as shown in Scheme 1 . Subsequent removal of diisopropyl L-malate with $\mathrm{MeOH}$ and $\mathrm{Et}_{3} \mathrm{~N}$ gave $N$-aryl phenylglycinate $(R)-3$ in $71 \%$ yield with 89:11 enantiomeric ratio (er). ${ }^{3 a, c}$ The yield and stereoselectivity of the substitution imply that $\alpha$-bromo stereogenic center is configurationally labile with respect to the rate of substitution and $(\alpha R S)-\mathbf{1}$ is dynamically resolved under the
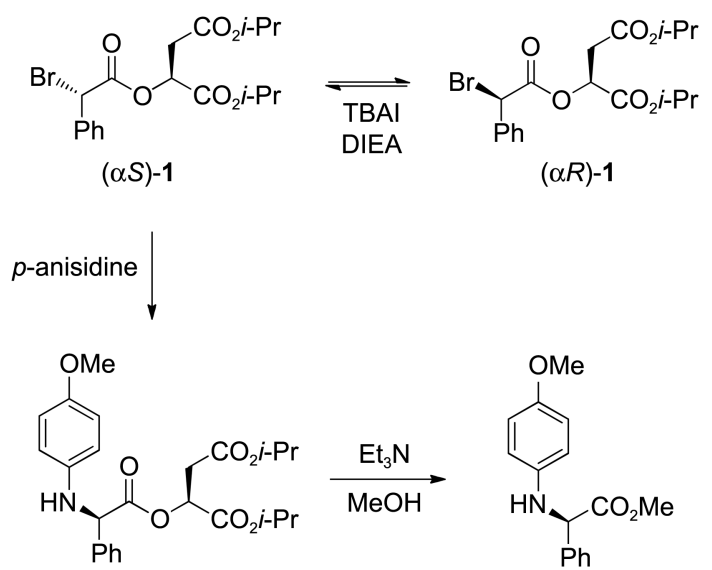

$(\alpha R)-2 \quad 90: 10 \mathrm{dr}$

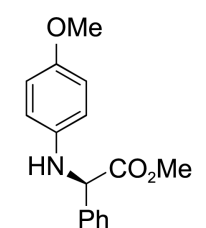

(R)-3 89:11 er
Scheme 1. Nucleophilic substitution with $p$-anisidine. reaction condition.

In order to understand the effect of additives and reaction pathway, we carried out a series of reactions as shown in Table 1. In the absence of both TBAI and DIEA, the substitution of 1 was very slow to provide 2 with 71:29 dr in $27 \%$ yield after $12 \mathrm{~h}$ (entry 1). Also, the rate of the substitution was substantially decreased in the absence of DIEA to provide 2 in 47\% yield with 90:10 dr, whereas the reaction in the absence of TBAI gave 2 with both lower yield and stereoselectivity (entries 2-3). The results in entries 1-3 show the importance of the presence of halide ion and base for sufficient rate acceleration and selectivity. When the mixture of two diastereomers of $\mathbf{1}$ (70:30 dr) was allowed to reach thermodynamic equilibrium in the presence of TBAI and DIEA, the diastereomeric ratio of recovered 1 was analyzed by ${ }^{1} \mathrm{H}$ NMR, and determined to be 51:49 (entry 4). The result implies that $\alpha$-bromo phenylacetate $\mathbf{1}$ is configurationally labile under the reaction condition and the thermodynamic stabilities of two diastereomers are almost same, ruling out dynamic thermodynamic resolution as a primary pathway. ${ }^{2}$ In the reaction of 1 (75:25 dr) with $p$-anisidine, DIEA and

Table 1. Dynamic kinetic resolution of $\alpha$-bromo phenylacetate 1

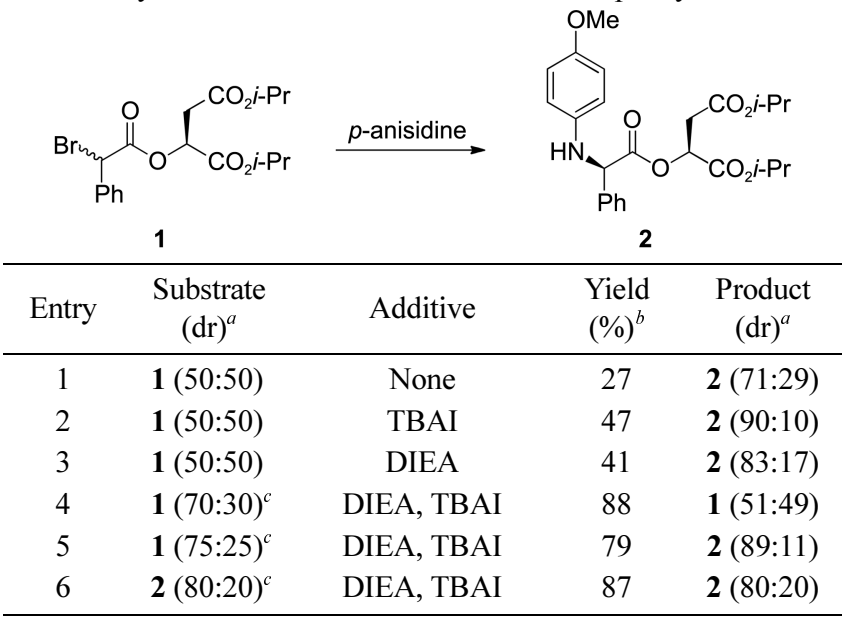

${ }^{a}$ The drs are determined by ${ }^{1} \mathrm{H}$ NMR. ${ }^{b}$ Isolated yields after $12 \mathrm{~h} .{ }^{c}$ The diastereomeric mixtures are prepared by column chromatography with fractional collection. 
TBAI as shown in entry 5, almost same dr of $2(89: 11 \mathrm{dr})$ was observed as in the reaction of $\mathbf{1}$ of 50:50 dr shown in Scheme 1. Thus, the dr of product $\mathbf{2}$ is not dependent on the starting ratio of two diastereomers of $\mathbf{1}$. When the configurational stability of $\mathbf{2}(80: 20 \mathrm{dr})$ under the reaction condition was examined by the treatment with $p$-anisidine (1.5 equiv), TBAI (1.0 equiv) and DIEA (1.0 equiv) in $\mathrm{CH}_{2} \mathrm{Cl}_{2}$ for $12 \mathrm{~h}$, no epimerization was detected by ${ }^{1} \mathrm{H}-\mathrm{NMR}$, which can rule out the possibility of epimerization after the nucleophilic substitution reaction (entry 6). To summarize the results in Table 1, we have found that the epimerization promoted by TBAI and DIEA is sufficiently fast with respect to the rate of substitution and the primary pathway of the asymmetric induction is a dynamic kinetic resolution.

The scope of the dynamic kinetic resolution has been investigated with various substrates and arylamines as shown in Table 2. The treatment of $\alpha$-bromo propanoate 4 with $p$ anisidine (1.5 equiv) in $\mathrm{CH}_{2} \mathrm{Cl}_{2}$ for $12 \mathrm{~h}$ at room temperature gave $\mathrm{N}$-aryl amino ester 6 in $60 \%$ yield with $83: 17 \mathrm{dr}$ (entry 1). Under the same reaction condition, the reactions of $\alpha$ bromo butanoate 5 gave amino ester 7 with 85:15 dr (entry 2). The reactions of $\mathbf{1}$ and $\mathbf{4}$ with $o$-anisidine as a nucleophile provided $N$-aryl amino esters 8 and $\mathbf{9}$ with slightly lower stereoselectivities compared to the reactions with $p$-anisidine (entries 3-4). In the reactions with two different aminoanthracenes, $N$-anthracenyl amino ester 10 of 86:14 dr was obtained with 1-aminoanthracene (entry 5), whereas 2-aminoanthracene gave slightly higher stereoselectivities (entry 6).

In the context of our continuing investigation on the stereoselective preparation of flavonoid derivatives and their activity studies, ${ }^{4}$ we have attempted to synthesize enantioenriched $N$-alkylated aminoflavones $\mathbf{1 2 - 1 4}$ as shown in entries 7-9. When $\alpha$-bromo phenylacetate 1 was treated with 6-aminoflavone, DIEA and TBAI for $12 \mathrm{~h}$, the substitution provided aminoflavone derivative 12 in $74 \%$ yield with 87:13 dr. Analogous to the reactions of $\mathbf{4}$ with $p$-anisidine and $o$-anisidine, the reaction of $\mathbf{4}$ with 6-aminoflavone gave lower selectivity of 82:18 dr (entries 7-8). Also, the substitution of 1 with 7 -aminoflavone provided aminoflavone derivative 14 in $64 \%$ yield with $83: 17 \mathrm{dr}$ under the same condition (entry 9) and the reactions of $\mathbf{4}$ with 7 -aminoflavone did not provide the substituted product. Thus, limited results in Table 2 indicate that $\alpha$-substituent of substrate and substituents of arylamine have significant effects on the stereoselectivity of nucleophilic substitution.

With the identification of diisopropyl L-malate as an effective and convenient stereocontrolling element for the reactions of $\alpha$-bromo acetates with various arylamines, we examined the dynamic kinetic resolution in substitutions with various 1,2-diaminobenzene nucleophiles for asymmetric syntheses of dihydroquinoxalinones as shown in Table 3. Dihydroquinoxalinone structural cores are of interest as important pharmacophores in many biologically active compounds and there is growing interest in the preparation of them. ${ }^{5}$ When $\alpha$-bromo phenylacetate $\mathbf{1}$ was treated with 1,2-phenylenediamine, TBAI and DIEA in $\mathrm{CH}_{2} \mathrm{Cl}_{2}$ for $24 \mathrm{~h}$ at room temperature, the substitution and following spon-
Table 2. Substitutions with various arylamine nucleophiles

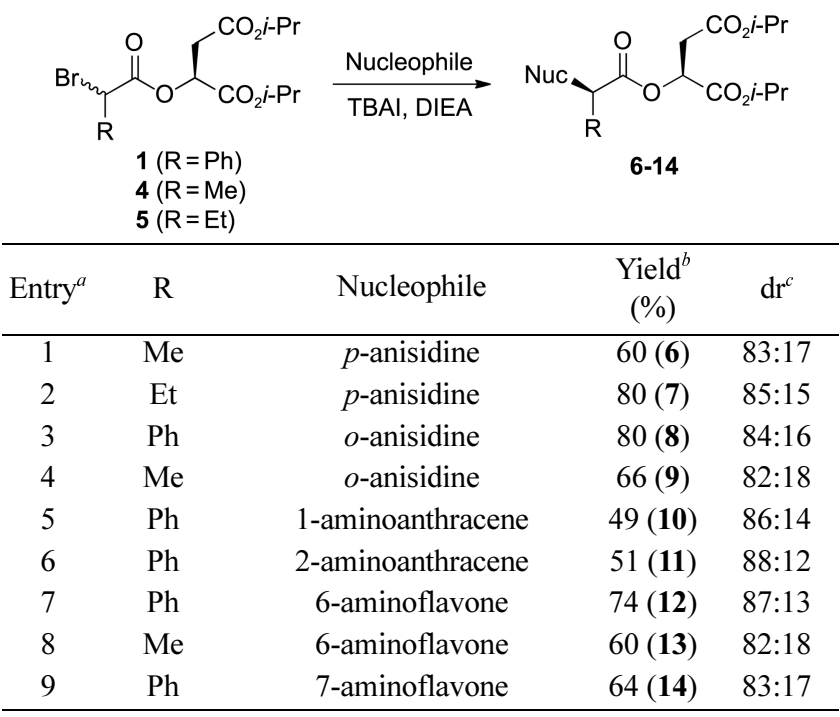

${ }^{a}$ All reactions were carried out in $\mathrm{CH}_{2} \mathrm{Cl}_{2} \cdot{ }^{b}$ Isolated yields. ${ }^{c}$ The drs are determined by ${ }^{1} \mathrm{H}$ NMR of reaction mixture.

taneous cyclization gave 3-phenyl dihydroquinoxalinone $\mathbf{1 5}$ in $85 \%$ yield with $88: 12$ er (entry 1). Notably, treatment of $\alpha$-bromo ester $\mathbf{1}$ with 2,3-diaminotoluene that is non-symmetric diaminobenzene nucleophile gave 3-phenyl-8-methyl dihydroquinoxalinone (16) as a major product with $84: 16$ er and no trace of the regioisomer was detected (entry 2). The regioselectivity suggests significantly different reactivities of two amino groups in the reaction of $\alpha$-bromo phenylacetate $\mathbf{1}$. The sterically less hindered amino group of the nucleophile is more reactive than the amino group with two ortho-substituents. The regiochemistry of $\mathbf{1 6}$ was assigned by comparison to the ${ }^{1} \mathrm{H}-\mathrm{NMR}$ of authentic material individually prepared. ${ }^{3 \mathrm{~b}}$ Treatment of $\mathbf{1}$ with 4,5-dimethyl-o-phenylenediamine, TBAI and DIEA for $24 \mathrm{~h}$ at room temperature gave 3-phenyl dihydroquinoxalinone (18) in 79\% yield with $88: 12$ er (entry 4), whereas the reactions of $\alpha$-bromo butanoate 5 with 4,5-dimethyl-o-phenylenediamine took place to afford 3-ethyl substituted dihydroquinoxalinones $\mathbf{1 7}$ with lower stereoselectivity of 78:22 er (entry 3). When the reaction of 1 with 1,2-phenylenediamine were carried out in $\mathrm{CH}_{3} \mathrm{CN}, 15$ was obtained with lower yield (40\%) and lower enantioselectivity (81:19 er) compared to the reaction in $\mathrm{CH}_{2} \mathrm{Cl}_{2}$.

In this paper, we report that diisopropyl L-malate is a new effective chiral auxiliary for dynamic kinetic resolution of $\alpha$ bromo esters in nucleophilic substitution with arylamines. The methodology can provide a general procedure for asymmetric syntheses of various $N$-aryl $(R)$-amino acid derivatives. In addition, the substitution with 1,2-diaminobenzene and subsequent spontaneous cyclization can provide an efficient method for asymmetric syntheses of dihydroquinoxalinones. We have found that the stereoselectivity of the substitution depends critically on the structure of arylamine and $\alpha$ substituent of substrate. Simple and easy procedure in obtaining optically active $N$-aryl amino esters suggests that diisopropyl L-malate-mediated nucleophilic substitution 
Table 3. Asymmetric syntheses of dihydroquinoxalinones

Entry ${ }^{a}$

${ }^{a}$ All reactions were carried out in $\mathrm{CH}_{2} \mathrm{Cl}_{2} .{ }^{b}$ Isolated yields after $24 \mathrm{~h}$. ${ }^{c}$ The ers are determined by CSP-HPLC (Chiralcel OJ-H column).

should be further developed.

\section{Experimental}

General Procedure for the Asymmetric Nucleophilic Substitution via Dynamic Kinetic Resolution. To a solution of $\alpha$-bromo ester $(\mathbf{1}, \mathbf{4}$ and $\mathbf{5})$ in $\mathrm{CH}_{2} \mathrm{Cl}_{2}(c a .0 .1 \mathrm{M})$ at room temperature were added DIEA (1.0 equiv), TBAI (1.0 equiv) and an arylamine nucleophile (1.5 equiv). After the resulting reaction mixture was stirred at room temperature for 12-24 h, the solvent was evaporated and the crude material was purified by column chromatography to give a $\alpha$-amino ester. The drs of $\mathbf{2}$ and $\mathbf{6 - 1 4}$ were determined by ${ }^{1} \mathrm{H}$ NMR integration of hydrogens of two diastereomers and the ers of 3 and 15-18 were determined by chiral stationary phase HPLC.

2-(p-Methoxyanilino)-(R)-phenylacetic Acid L-Diisopropyl Malate Ester (2). A yellow oil was obtained in $81 \%$ yield. ${ }^{1} \mathrm{H} \mathrm{NMR}\left(\mathrm{CDCl}_{3}, 400 \mathrm{MHz}\right.$, major diastereomer $) 7.48$ $(\mathrm{m}, 2 \mathrm{H}), 7.32(\mathrm{~m}, 3 \mathrm{H}), 6.73(\mathrm{~d}, J=7.2 \mathrm{~Hz}, 2 \mathrm{H}), 6.56(\mathrm{~d}, J=$ $7.2 \mathrm{~Hz}, 2 \mathrm{H}), 5.43(\mathrm{t}, J=6.0 \mathrm{~Hz}, 1 \mathrm{H}), 5.11(\mathrm{~s}, 1 \mathrm{H}), 5.07(\mathrm{~m}$, $1 \mathrm{H}), 4.78(\mathrm{~m}, 1 \mathrm{H}), 3.71(\mathrm{~s}, 3 \mathrm{H}), 2.74(\mathrm{~d}, J=6.4 \mathrm{~Hz}, 2 \mathrm{H})$, 1.28-1.01 (m, 12H); ${ }^{13} \mathrm{C}$ NMR $\left(\mathrm{CDCl}_{3}, 100 \mathrm{MHz}\right.$, major diastereomer) 171.1, 168.1, 167.9, 152.5, 140.1, 137.3, 128.9, $128.4,127.5,114.8,114.7,69.9,69.5,68.7,61.5,55.7,36.4$, $21.8,21.6$. For removal of chiral auxiliary, the mixture of 2 and $\mathrm{Et}_{3} \mathrm{~N}$ (10 equiv) in methanol $(0.05 \mathrm{M})$ was stirred at room temperature for a day. The solvent was evaporated and the crude material was purified by column chromatography to give $(R)-3$ in $71 \%$ yield. ${ }^{1} \mathrm{H}$ NMR $\left(\mathrm{CDCl}_{3}, 400 \mathrm{MHz}\right)$ 7.48-7.25 (m, 5H), $6.69(\mathrm{~d}, J=8.9 \mathrm{~Hz}, 2 \mathrm{H}), 6.52(\mathrm{~d}, J=8.9$ $\mathrm{Hz}, 2 \mathrm{H}), 5.00(\mathrm{~s}, 1 \mathrm{H}), 4.67$ (br, 1H), $3.72(\mathrm{~s}, 3 \mathrm{H}), 3.67$ (s, $3 \mathrm{H})$. The spectral data of $\mathbf{3}$ were identical to those of the authentic material reported in ref. 3. Chiral HPLC: 87:13 er,
$t_{\mathrm{R}}(R)$-major enantiomer, $67.2 \mathrm{~min} ; t_{\mathrm{R}}(S)$-minor enantiomer, $76.3 \mathrm{~min}$ (Chiralcel OJ-H column; 20\% 2-propanol in hexane; $0.5 \mathrm{~mL} / \mathrm{min}$ ).

2-(p-Methoxyanilino)-(R)-Propanoic Acid L-Diisopropyl Malate Ester (6). A yellow oil was obtained in $60 \%$ yield. ${ }^{1} \mathrm{H}$ NMR $\left(\mathrm{CDCl}_{3}, 400 \mathrm{MHz}\right.$, major diastereomer) $6.74(\mathrm{~d}, J$ $=7.2 \mathrm{~Hz}, 2 \mathrm{H}), 6.60(\mathrm{~d}, J=7.2 \mathrm{~Hz}, 2 \mathrm{H}), 5.44(\mathrm{~m}, 1 \mathrm{H}), 5.05$ (m, 1H), $4.97(\mathrm{~m}, 1 \mathrm{H}), 4.14(\mathrm{q}, J=6.8 \mathrm{~Hz}, 1 \mathrm{H}), 3.73(\mathrm{~s}, 3 \mathrm{H})$, $2.80(\mathrm{~m}, 2 \mathrm{H}), 1.49(\mathrm{~d}, J=6.8 \mathrm{~Hz}, 3 \mathrm{H}), 1.21(\mathrm{~m}, 12 \mathrm{H}) ;{ }^{13} \mathrm{C}$ NMR $\left(\mathrm{CDCl}_{3}, 100 \mathrm{MHz}\right.$, major diastereomer) $173.8,168.4$, $168.0,152.8,140.6,115.1,114.9,69.7,68.9,68.8,55.7$, $52.9,36.5,21.7,21.6,19.0$.

2-(p-Methoxyanilino)-(R)-Butanoic Acid L-Diisopropyl Malate Ester (7). A pale yellow oil was obtained in $80 \%$ yield. ${ }^{1} \mathrm{H} \mathrm{NMR}\left(\mathrm{CDCl}_{3}, 400 \mathrm{MHz}\right.$, major diastereomer $) 6.75$ (d, $J=7.2 \mathrm{~Hz}, 2 \mathrm{H}), 6.61$ (d, $J=7.2 \mathrm{~Hz}, 2 \mathrm{H}), 5.44$ (t, $J=6.4$ Hz, 1H), $5.04(\mathrm{~m}, 1 \mathrm{H}), 4.97(\mathrm{~m}, 1 \mathrm{H}), 4.01(\mathrm{t}, J=6.4 \mathrm{~Hz}$, $1 \mathrm{H}), 3.73(\mathrm{~s}, 3 \mathrm{H}), 2.80(\mathrm{~d}, J=6.4 \mathrm{~Hz}, 2 \mathrm{H}), 1.91-1.78(\mathrm{~m}$, $2 \mathrm{H}), 1.22(\mathrm{~m}, 12 \mathrm{H}), 1.04(\mathrm{t}, J=7.6 \mathrm{~Hz}, 3 \mathrm{H}) ;{ }^{13} \mathrm{C} \mathrm{NMR}$ $\left(\mathrm{CDCl}_{3}, 100 \mathrm{MHz}\right.$, major diastereomer) 173.4, 168.5, 168.1, $152.7,140.8,115.3,114.9,69.9,68.9,68.6,58.8,55.7,36.6$, 26.2, 21.7, 21.5, 10.1 .

2-(o-Methoxyanilino)-(R)-Phenylacetic Acid L-Diisopropyl Malate Ester (8). A yellow oil was obtained in $80 \%$ yield. ${ }^{1} \mathrm{H} \mathrm{NMR}\left(\mathrm{CDCl}_{3}, 400 \mathrm{MHz}\right.$, major diastereomer $) 7.51$ $(\mathrm{m}, 2 \mathrm{H}), 7.33(\mathrm{~m}, 3 \mathrm{H}), 6.73(\mathrm{~m}, 3 \mathrm{H}), 6.41(\mathrm{~m}, 1 \mathrm{H}), 5.43(\mathrm{t}, J$ $=6.0 \mathrm{~Hz}, 1 \mathrm{H}), 5.36(\mathrm{~d}, J=6.0 \mathrm{~Hz}, 1 \mathrm{H}), 5.16(\mathrm{~d}, J=6.0 \mathrm{~Hz}$, $1 \mathrm{H}), 5.07(\mathrm{~m}, 1 \mathrm{H}), 4.79(\mathrm{~m}, 1 \mathrm{H}), 3.86(\mathrm{~s}, 3 \mathrm{H}), 2.74(\mathrm{~d}, J=$ $6.4 \mathrm{~Hz}, 2 \mathrm{H}), 1.25-1.00(\mathrm{~m}, 12 \mathrm{H}) ;{ }^{13} \mathrm{C}$ NMR $\left(\mathrm{CDCl}_{3}, 100\right.$ MHz, major diastereomer) 170.8, 168.1, 167.9, 147.0, 137.2, $135.9,128.8,128.3,127.5,121.1,117.5,110.7,109.5,69.8$, 69.5, 68.7, 60.6, 55.4, 36.4, 21.6, 21.5.

2-(o-Methoxyanilino)-(R)-Propanoic Acid L-Diisopropyl Malate Ester (9). A pale yellow oil was obtained in $66 \%$ yield. ${ }^{1} \mathrm{H}$ NMR $\left(\mathrm{CDCl}_{3}, 400 \mathrm{MHz}\right.$, major diastereomer) 6.84-6.55 (m, 4H), $5.45(\mathrm{~m}, 1 \mathrm{H}), 5.06(\mathrm{~m}, 1 \mathrm{H}), 4.96(\mathrm{~m}, 1 \mathrm{H})$, $4.65(\mathrm{br}, 1 \mathrm{H}), 4.21(\mathrm{~m}, 1 \mathrm{H}), 3.85(\mathrm{~s}, 3 \mathrm{H}), 2.81(\mathrm{~m}, 2 \mathrm{H}), 1.54$ $(\mathrm{d}, J=6.8 \mathrm{~Hz}, 3 \mathrm{H}), 1.22(\mathrm{~m}, 12 \mathrm{H}) ;{ }^{13} \mathrm{C} \mathrm{NMR}\left(\mathrm{CDCl}_{3}, 100\right.$ MHz, major diastereomer) 173.4, 168.5, 168.1, 152.7, 140.8, $115.3,114.9,69.7,69.0,68.8,55.4,51.6,36.5,21.7,21.6$, 18.9 .

2-(1-Anthracenylamino)-(R)-Phenylacetic Acid L-Diisopropyl Malate Ester (10). A yellow oil was obtained in $49 \%$ yield. ${ }^{1} \mathrm{H} \mathrm{NMR}\left(\mathrm{CDCl}_{3}, 400 \mathrm{MHz}\right.$, major diastereomer) $8.55(\mathrm{~s}, 1 \mathrm{H}), 8.35(\mathrm{~s}, 1 \mathrm{H}), 8.06(\mathrm{~m}, 1 \mathrm{H}), 7.98(\mathrm{~m}, 1 \mathrm{H}), 7.62-$ $7.21(\mathrm{~m}, 9 \mathrm{H}), 6.32(\mathrm{~d}, J=7.2 \mathrm{~Hz}, 1 \mathrm{H}), 5.51(\mathrm{t}, J=6.0 \mathrm{~Hz}$, $1 \mathrm{H}), 5.43(\mathrm{~s}, 1 \mathrm{H}), 5.10(\mathrm{~m}, 1 \mathrm{H}), 4.78(\mathrm{~m}, 1 \mathrm{H}), 2.80(\mathrm{~d}, J=$ $6.0 \mathrm{~Hz}, 2 \mathrm{H}), 1.25(\mathrm{~m}, 6 \mathrm{H}), 1.10(\mathrm{~d}, J=6.0 \mathrm{~Hz}, 3 \mathrm{H}), 1.02(\mathrm{~d}$, $J=6.0 \mathrm{~Hz}, 3 \mathrm{H}) ;{ }^{13} \mathrm{C} \mathrm{NMR}\left(\mathrm{CDCl}_{3}, 100 \mathrm{MHz}\right.$, major diastereomer) 171.0, 168.0, 167.9, 140.5, 136.8, 131.6, 131.0, $129.0,128.5,127.8,127.4,126.7,125.9,125.6,125.2$, 118.9, 118.4, 103.6, 70.0, 69.8, 68.9, 60.8, 36.4, 31.0, 21.7.

2-(2-Anthracenylamino)-(R)-Phenylacetic Acid L-Diisopropyl Malate Ester (11). A yellow oil was obtained in $51 \%$ yield. ${ }^{1} \mathrm{H} \mathrm{NMR}\left(\mathrm{CDCl}_{3}, 400 \mathrm{MHz}\right.$, major diastereomer) $8.22(\mathrm{~s}, 1 \mathrm{H}), 8.05(\mathrm{~s}, 1 \mathrm{H}), 7.83(\mathrm{~m}, 3 \mathrm{H}), 7.58(\mathrm{~m}, 2 \mathrm{H}), 7.36$ (m, 5H), $7.03(\mathrm{~m}, 1 \mathrm{H}), 6.73(\mathrm{~s}, 1 \mathrm{H}), 5.47(\mathrm{t}, J=6.0 \mathrm{~Hz}, 1 \mathrm{H})$, 
$5.34(\mathrm{~s}, 1 \mathrm{H}), 5.08(\mathrm{~m}, 2 \mathrm{H}), 4.77(\mathrm{~m}, 1 \mathrm{H}), 2.77(\mathrm{~d}, J=6.4 \mathrm{~Hz}$, 2H), 1.29-1.00 (m, 12H); ${ }^{13} \mathrm{C} \mathrm{NMR}\left(\mathrm{CDCl}_{3}, 100 \mathrm{MHz}\right.$, major diastereomer) 171.0, 168.0, 167.9, 142.6, 136.8, 133.2, 132.4, 129.7, 129.5, 129.0, 128.6, 128.2, 127.6, 127.4, 126.1, 125.3, $123.8,123.1,120.1,103.3,70.0,69.7,68.8,60.9,36.4,31.6$, 21.6, 21.5 .

2-[(4-Oxo-2-phenyl-4H-chromem-6-yl)amino]phenylacetic Acid L-Diisopropyl Malate Ester (12). A colorless oil was obtained in $74 \%$ yield. ${ }^{1} \mathrm{H} \mathrm{NMR}\left(\mathrm{CDCl}_{3}, 400 \mathrm{MHz}\right.$, major diastereomer) $7.87(\mathrm{~m}, 2 \mathrm{H}), 7.53-7.23(\mathrm{~m}, 10 \mathrm{H}), 7.02$ $(\mathrm{m}, 1 \mathrm{H}), 6.74(\mathrm{~s}, 1 \mathrm{H}), 5.44(\mathrm{~m}, 1 \mathrm{H}), 5.32(\mathrm{~d}, J=6.0 \mathrm{~Hz}, 1 \mathrm{H})$, $5.19(\mathrm{~d}, J=6.0 \mathrm{~Hz}, 1 \mathrm{H}), 5.09(\mathrm{~m}, 1 \mathrm{H}), 4.81(\mathrm{~m}, 1 \mathrm{H}), 2.76(\mathrm{~d}$, $J=6.4 \mathrm{~Hz}, 2 \mathrm{H}), 1.24(\mathrm{~d}, J=6.4 \mathrm{~Hz}, 3 \mathrm{H}), 1.20(\mathrm{~d}, J=6.4 \mathrm{~Hz}$, $3 \mathrm{H}), 1.12(\mathrm{~d}, J=6.4 \mathrm{~Hz}, 3 \mathrm{H}), 1.05(\mathrm{~d}, J=6.4 \mathrm{~Hz}, 3 \mathrm{H}) ;{ }^{13} \mathrm{C}$ $\mathrm{NMR}\left(\mathrm{CDCl}_{3}, 100 \mathrm{MHz}\right.$, major diastereomer) 178.3, 170.6, $168.0,167.7,162.7,149.8,143.3,136.4,132.1,131.3$, $129.0,128.9,128.6,127.3,126.2,124.7,121.1,119.1$, 106.7, 105.7, 70.0, 69.9, 68.8, 60.4, 36.2, 29.7, 21.6.

2-[(4-Oxo-2-phenyl-4H-chromem-6-yl)amino]propanoic Acid L-Diisopropyl Malate Ester (13). A colorless oil was obtained in $60 \%$ yield. ${ }^{1} \mathrm{H} \mathrm{NMR}\left(\mathrm{CDCl}_{3}, 400 \mathrm{MHz}\right.$, major diastereomer) $7.91(\mathrm{~m}, 2 \mathrm{H}), 7.52(\mathrm{~m}, 3 \mathrm{H}), 7.44(\mathrm{~d}, J=8.8$ $\mathrm{Hz}, 1 \mathrm{H}), 7.31(\mathrm{~m}, 1 \mathrm{H}), 7.04(\mathrm{dd}, J=2.8$ and $8.8 \mathrm{~Hz}, 1 \mathrm{H})$, $6.78(\mathrm{~s}, 1 \mathrm{H}), 5.46(\mathrm{~m}, 1 \mathrm{H}), 5.05(\mathrm{~m}, 1 \mathrm{H}), 4.98(\mathrm{~m}, 1 \mathrm{H}), 4.41$ $(\mathrm{m}, 1 \mathrm{H}), 2.86(\mathrm{~m}, 2 \mathrm{H}), 1.55(\mathrm{~d}, J=6.4 \mathrm{~Hz}, 3 \mathrm{H}), 1.23(\mathrm{~m}$, $12 \mathrm{H}) ;{ }^{13} \mathrm{C} \mathrm{NMR}\left(\mathrm{CDCl}_{3}, 100 \mathrm{MHz}\right.$, major diastereomer) $178.4,173.0,168.4,167.9,162.8,150.0,144.0,132.1$, $131.3,129.0,126.2,124.8,121.3,119.2,106.7,105.3,69.9$, 69.3, 68.8, 51.9, 36.4, 21.8, 21.6, 18.7 .

2-[(4-Oxo-2-phenyl-4H-chromem-7-yl)amino]phenylacetic Acid L-Diisopropyl Malate Ester (14). A colorless oil was obtained in $64 \%$ yield. ${ }^{1} \mathrm{H} \mathrm{NMR}\left(\mathrm{CDCl}_{3}, 400 \mathrm{MHz}\right.$, major diastereomer) $7.99(\mathrm{~d}, J=8.8 \mathrm{~Hz}, 1 \mathrm{H}), 7.81(\mathrm{~m}, 2 \mathrm{H})$, $7.53-7.41(\mathrm{~m}, 8 \mathrm{H}), 6.71(\mathrm{~m}, 1 \mathrm{H}), 6.69(\mathrm{~s}, 1 \mathrm{H}), 6.51(\mathrm{~d}, J=$ $2.1 \mathrm{~Hz}, 1 \mathrm{H}), 5.48(\mathrm{~m}, 2 \mathrm{H}), 5.28(\mathrm{~d}, J=5.6 \mathrm{~Hz}, 1 \mathrm{H}), 5.09(\mathrm{~m}$, $1 \mathrm{H}), 4.79(\mathrm{~m}, 1 \mathrm{H}), 2.79(\mathrm{~d}, J=6.0 \mathrm{~Hz}, 2 \mathrm{H}), 1.22(\mathrm{~m}, 6 \mathrm{H})$, $1.12(\mathrm{~d}, J=6.0 \mathrm{~Hz}, 3 \mathrm{H}), 1.04$ (d, $J=6.0 \mathrm{~Hz}, 3 \mathrm{H}) ;{ }^{13} \mathrm{C} \mathrm{NMR}$ $\left(\mathrm{CDCl}_{3}, 100 \mathrm{MHz}\right.$, major diastereomer) $177.7,170.0$, 167.9, 167.7, 162.4, 158.4, 150.0, 135.7, 132.1, 131.1, 129.2, 128.9, $128.8,127.3,126.9,126.1,115.7,113.4,107.4,98.3,70.2$, 69.9, 68.9, 60.1, 36.3, 31.0, 21.6.

3-Phenyl-3,4-dihydro-1,4-quinoxalin-2-one (15). A white solid was obtained in $85 \%$ yield. ${ }^{1} \mathrm{H} \mathrm{NMR}\left(\mathrm{CDCl}_{3}, 400\right.$ $\mathrm{MHz}) 7.95$ (br, 1H), 7.43-6.70 (m, 9H), 5.08 (s, 1H), 4.28 (br, $1 \mathrm{H})$. The spectral data were identical to those of the authentic material reported in ref. 3. Chiral HPLC: 88:12 er, $t_{\mathrm{R}}(R)$-major enantiomer, $39.3 \mathrm{~min}$; $t_{\mathrm{R}}(S)$-minor enantiomer, $44.0 \mathrm{~min}$ (Chiralcel OJ-H column; 20\% 2-propanol in hexane; $0.5 \mathrm{~mL} / \mathrm{min})$.

8-Methyl-3-phenyl-3,4-dihydro-1,4-quinoxalin-2-one (16). A pale yellow oil was obtained in $71 \%$ yield. ${ }^{1} \mathrm{H}$ NMR $\left(\mathrm{CDCl}_{3}, 400 \mathrm{MHz}\right) 8.45$ (br, 1H), 7.40-7.23 (m, 5H), 6.826.55 (m, 3H), 5.00 (s, 1H), $4.30(\mathrm{br}, 1 \mathrm{H}), 2.16(\mathrm{~s}, 3 \mathrm{H}) ;{ }^{13} \mathrm{C}$
$\mathrm{NMR}\left(\mathrm{CDCl}_{3}, 100 \mathrm{MHz}\right) 167.6,139.3,133.5,131.4,129.2$, 127.6, 125.9, 124.0, 121.7, 119.2, 114.2, 61.0, 17.0. Chiral HPLC: $84: 16 \mathrm{er}, t_{\mathrm{R}}(R)$-major enantiomer, $35.8 \mathrm{~min} ; t_{R}(S)$ minor enantiomer, 42.0 min (Chiralcel OJ-H column; 20\% 2-propanol in hexane; $0.5 \mathrm{~mL} / \mathrm{min}$ ).

6,7-Dimethyl-3-ethyl-3,4-dihydro-1,4-quinoxalin-2-one (17). A pale yellow oil was obtained in $69 \%$ yield. ${ }^{1} \mathrm{H}$ NMR $\left(\mathrm{CDCl}_{3}, 400 \mathrm{MHz}\right) 8.44(\mathrm{br}, 1 \mathrm{H}), 6.51(\mathrm{~s}, 1 \mathrm{H}), 6.49(\mathrm{~s}, 1 \mathrm{H})$, $3.80(\mathrm{~m}, 2 \mathrm{H}), 2.15(\mathrm{~s}, 6 \mathrm{H}), 1.79(\mathrm{~m}, 2 \mathrm{H}), 1.02(\mathrm{t}, J=7.4 \mathrm{~Hz}$, $3 \mathrm{H})$. The spectral data were identical to those of the authentic material reported in ref. 3. Chiral HPLC: 78:22 er, $t_{\mathrm{R}}(R)$-major enantiomer, $19.3 \mathrm{~min} ; t_{\mathrm{R}}(S)$-minor enantiomer, $26.6 \mathrm{~min}$ (Chiralcel OJ-H column; 15\% 2-propanol in hexane; $0.5 \mathrm{~mL} / \mathrm{min})$.

6,7-Dimethyl-3-phenyl-3,4-dihydro-1,4-quinoxalin-2-one (18). A white solid was obtained in $79 \%$ yield. ${ }^{1} \mathrm{H}$ NMR $\left(\mathrm{CDCl}_{3}, 400 \mathrm{MHz}\right) 8.44$ (br, 1H), 7.41-7.28 (m, 5H), $6.50(\mathrm{~s}$, $2 \mathrm{H}), 5.01(\mathrm{~s}, 1 \mathrm{H}), 4.11(\mathrm{br}, 1 \mathrm{H}), 2.15$ (s, 3H), 2.14 (s, 3H). The spectral data were identical to those of the authentic material reported in ref. 3. Chiral HPLC: $88: 12 \mathrm{er}, t_{\mathrm{R}}(R)-$ major enantiomer, $34.1 \mathrm{~min} ; t_{\mathrm{R}}(S)$-minor enantiomer, 45.8 min (Chiralcel OJ-H column; 20\% 2-propanol in hexane; 0.5 $\mathrm{mL} / \mathrm{min})$.

Acknowledgments. This work was supported by the Priority Research Centers Program through the National Research Foundation of Korea (2009-0093824) and a National Research Foundation of Korea Grant funded by the Korean Government (2009-0089650).

\section{References and Notes}

1. (a) Langer, K.; Mattay, J. J. Org. Chem. 1995, 60, 7256. (b) Thiam, M.; Chastrette, F. Tetrahedron Lett. 1990, 31, 1429.

2. Park, Y. S. Tetrahedron: Asymmetry 2009, 43, 8253, and references therein.

3. The absolute configurations of $(R)-\mathbf{3}$ and $(R)$-15-18 were determined by comparison of CSP-HPLC retention time with the reported values. (a) Kim, H. J.; Shin, E.-K.; Chang, J.-Y.; Kim, Y.; Park, Y. S. Tetrahedron Lett. 2005, 46, 4115. (b) Kim, Y.; Lee, M. H.; Choi, E. T.; No, E. S.; Park, Y. S. Heterocycles 2007, 71, 5. (c) Lee, Y. M.; Park, Y. S. Heterocycles 2009, 78, 2233. (d) Kim, Y.; Kang, K. H.; Choi, E. T.; Lee, M. H.; Park, Y. S. Bull. Korean Chem. Soc. 2007, 28, 325.

4. (a) Park, J.-H.; Lee, J.-W.; Paik, H.-D.; Cho, S. G.; Nah, S.-Y.; Park, Y. S.; Han, Y. S. Food Sci. Biotechnol. 2010, 19, 717. (b) Lee, E.R.; Kang, Y.-J.; Kim, H.-J.; Choi, H.-Y.; Kang, G.-H.; Kim, J.-H.; Kim, B.-W.; Jeong, H.-S.; Park, Y. S.; Cho, S.-G. J. Cell. Biochem. 2008, 104, 259. (c) Lee, E.-R.; Kang, Y.-J.; Choi, H.-Y.; Kang, G.H.; Kim, J.-H.; Kim, B.-W.; Han, Y.-S.; Nah, S.-Y.; Paik, H.-D.; Park, Y. S.; Cho, S.-G. Biol. Pharm. Bull. 2007, 30, 2394.

5. (a) Jiang, Q.; Jiang, D.; Jiang, Y.; Fu, H.; Zhao, Y. Synlett 2007, 1836. (b) Abraham, C.; Paull, D. H.; Scerba, M. T.; Grebinski, J. W.; Lectka, T. J. Am. Chem. Soc. 2006, 128, 13370. (c) Mahaney, P. E.; Webb, M. B.; Ye, F.; Sabatucci, J. P.; Steffan, R. J.; Chadwick, C. C.; Harnish, D. C.; Trybulski, E. J. Bioorg. Med. Chem. 2006, 14, 3455. 